\title{
Circularly Polarised Hexagonal Patch Antenna With Polygonal Slot for RFID Applications
}

\author{
Prakash K. C, Vinesh P. V, Vivek R, Mohammad Ameen, and Vasudevan K
}

\begin{abstract}
A compact single feed circularly polarized microstrip patch antenna for RFID applications is proposed. Antenna geometry includes a regular hexagon shaped patch with a polygonal slot embedded at the centre. The slot accounts for circular polarization and an area reduction of $22.5 \%$. It is fabricated on FR4 substrate with dielectric constant 4.4 and size $50 \mathrm{~mm} \times 50 \mathrm{~mm} \times 1.6 \mathrm{~mm}$. The measured results include $10 \mathrm{~dB}$ impedance bandwidth of $5.5 \%$ at the center frequency of 2.42 $\mathbf{G H z}$, a return loss of $32 \mathrm{~dB}$, minimum axial ratio of $1.82 \mathrm{~dB}$, axial ratio bandwidth of $7.5 \%$, gain of $4.9 \mathrm{dBi}$ with a broadside radiation characteristic for the RHCP antenna. These results are well in tune with the simulated results and the proposed design is suitable for RFID reader antenna applications.
\end{abstract}

Index terms - circularly polarized, hexagonal patch antenna, polygonal slot, RFID

\section{INTRODUCTION}

RFID based identification of objects and people have become highly popular. The RFID system generally consists of two basic components; the reader and the tag. The reader is a read or read/write device that uses an antenna to send a radio frequency electromagnetic field to the tag. The tag is the data carrying device located on the object being identified. RFID reader antennas widely use circularly polarized single feed microstrip patch antennas in order to overcome false identification of targeted objects [1]. Commonly used design of circularly polarized patch antennas either excite two orthogonal modes of a symmetrical patch (single coaxial feed) or patch antennas with two orthogonal feeds which are in quadrature. Various advantages of single feed circularly polarized antennas include reduced feeding circuit complexity, less weight, compact and preferred in situations where it is difficult to accommodate dual orthogonal feeds. The proposed single feed circularly polarized antenna is distinct due to several features. Perturbation methods are employed to bring circularly polarized radiation. Currently a wide variety of perturbation methods are there. The methods include, using a cross slot embedded on the radiating patch [2], truncating the corners of a square patch and inserting slits of different lengths at the edges of a square patch [3], the combination of two equal right angle

Manuscript received April 4, 2016; revised June 2, 2016.

This work has been supported by University Grants Commission, New Delhi, India.

Authors are with the Centre for Research in Electromagnetics and antennas, Dept. of Electronics, Cochin University of Science and Technology, Cochin, Kerala, India (E-mail: keyceepee@gmail.com). the slot antennas with equal perpendicular length on the ground plane fed by microstrip line [4], inserting an elliptical slot at centre of a circular patch [5], a circular patch with a rectangular slot oriented at $45^{0}$ [6], two asymmetric orthogonal slits positioned in the centre of the patch antenna [7], In the proposed antenna instead of an elliptical slot, irregular hexagonal slot is cut in a hexagonal patch. In this article the promising design of a single co- axially fed circular polarized microstrip antenna is

presented. The design strategy in this work is simultaneous control over frequency, axial ratio and input impedance and thereby achieving $\mathrm{CP}$ and matching conditions. This is achieved with the choice of shape for the central slot and the

feed point. The insertion of the slot is to i) produce circularly polarized radiation in the required band ii) reduce the size of the patch and iii) bring down resonant frequency. The choice of dimensions of the central irregular slot and the feed position ensures the splitting of the fundamental mode into degenerate modes and phase quadrature between these modes. These degenerate modes should have equal amplitude and exact phase quadrature to ensure circularly polarized radiation. A circular patch antenna with central elliptical slot is used to generate CP radiation in [5]. It is substantiated in Section IV that all the measured values of the proposed antenna in this work are superior to other similar works in literature. The dimensions of the patch and slot, feed point locations are optimized for good performance. Ansoft HFSS is used for the simulation and analysis of the structure. The antenna performance is evaluated using ZVB20 vector network analyzer.

The proposed RHCP antenna has got a $10 \mathrm{~dB}$ impedance bandwidth of $130 \mathrm{MHz}(2.355 \mathrm{GHz}$ to $2.485 \mathrm{GHz})$ and axial ratio bandwidth of $180 \mathrm{MHz}(2.32 \mathrm{GHz}$ to $2.5 \mathrm{GHz})$ and a gain of 4.9dBi. Though this band is usable for WLAN and Bluetooth applications, the obtained gain, axial ratio bandwidth and $80^{\circ}$ $3 \mathrm{~dB}$ beam width are promising factors for its appropriateness for active RFID reader antenna applications. However this gain is insufficient for long range applications, it can be enhanced by incorporating stacking concept.

Active RFID and passive RFID technologies are basically different technologies with substantially distinct capabilities. In terms of communication range, active RFID offers a range of more than $100 \mathrm{~m}$, while it is nearly $12 \mathrm{~m}$ for passive RFID systems. Active RFID collects signals from 1000s of tags by the reader while it is 100s in UHF passive RFID systems. Active RFID has got the ability to continuously monitor and record sensor input, whereas the passive RFID system has got the ability to read and transfer sensor values only when the tag is 
powered by the reader. Active RFID has got large read/write data storage $(128 \mathrm{~KB})$ with sophisticated data search and access capabilities, whereas the passive RFID has got small read/write data storage. Active RFID is capable of area monitoring, sophisticated cargo security applications and unconstrained asset movement. When considering the cost, this is less for UHF passive RFID.

The novelty of the proposed antenna is its shape, area reduction, compactness and the mode of generation of circularly polarized radiation. The authors have experimentally proved in [8] that when the patch shape is dodecagonal gain is slightly less $(4.82 \mathrm{dBi}-4.67 \mathrm{dBi})$, ARBW is $5.5 \%$ and when the shape is hexagonal with elliptical and circular slots the gain is $4.5 \mathrm{dBi}$ and $\mathrm{ARBW}$ is $5.8 \%$ [9]; both designed for the same resonant frequency $2.45 \mathrm{GHz}$.

The measured parameters of the antenna show its suitability for active RFID reader antenna applications.

\section{ANTENNA GEOMETRY}

The proposed antenna is fabricated on $50 \mathrm{~mm} \times 50 \mathrm{~mm}$ FR 4 substrate, with dielectric constant 4.4 , thickness $1.6 \mathrm{~mm}$ and a $\tan \delta=0.02$. It consists of regular hexagonal patch with an irregular polygonal slot.

\section{feed point for RHCP}

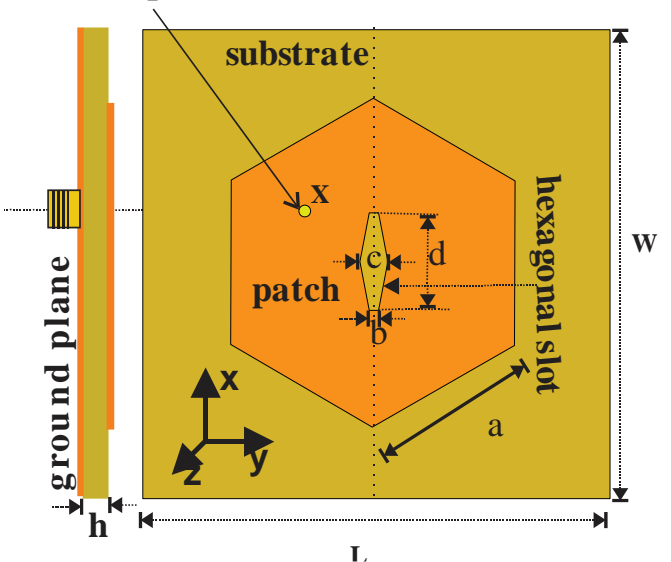

(a)

(b)

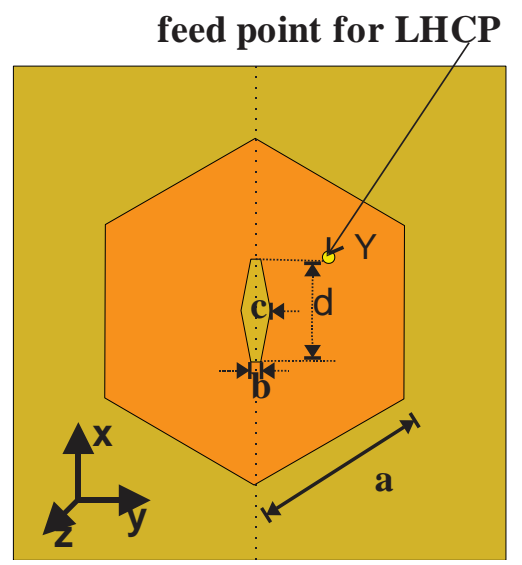

(c)

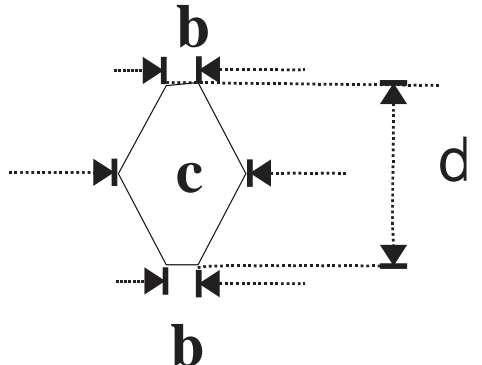

(d)

Fig. 1. Geometry of the proposed antenna.(a) side view (b) Top view of RHCP antenna (c) Top view of LHCP antenna. (d) central irregular hexagonal slot geometry, Guided wavelength $\lambda_{\mathrm{g}}=0.0604 \mathrm{~m}, \mathrm{a}=$ $17.244 \mathrm{~mm}\left(0.285 \lambda_{\mathrm{g}}\right) \mathrm{b}=1 \mathrm{~mm}\left(0.0166 \lambda_{\mathrm{g}}\right), \mathrm{c}=7 \mathrm{~mm}\left(0.116 \lambda_{\mathrm{g}}\right), \mathrm{d}=$ $11.4 \mathrm{~mm}\left(0.189 \lambda_{\mathrm{g}}\right), \mathrm{k}=1.62, \mathrm{~L}=\mathrm{W}=50 \mathrm{~mm}\left(0.8278 \lambda_{\mathrm{g}}\right) \cdot \mathrm{X}(21 \mathrm{~mm}, 19$ $\mathrm{mm}), \mathrm{Y}(21 \mathrm{~mm}, 31 \mathrm{~mm})$.

The patch is fabricated using copper material of thickness $0.035 \mathrm{~mm}$ on the top side of the substrate and the copper ground plane is of dimensions $50 \mathrm{~mm} \times 50 \mathrm{~mm} \times 0.035 \mathrm{~mm}$ on the bottom side of the substrate. As depicted in Figure 1, the irregular hexagonal slot is situated on the centre of the patch oriented along the $\mathrm{X}$ axis. The dimensions of the hexagonal slot are designated as i) b - the smaller $\mathrm{Y}$ axis dimension (top and bottom width), ii) $\mathrm{c}$ - the larger $\mathrm{Y}$ axis dimension (central width) and iii) $\mathrm{d}-$ the larger $\mathrm{X}$ axis dimension (height). These dimensions are such that $\mathrm{b}<\mathrm{c}<\mathrm{d}$. The ratio ' $\mathrm{k}$ ' is defined as ratio of ' $d$ ' to ' $c$ ' with ' $b$ ' constant. In order to generate circularly polarized waves, the simplest approach is to excite two equal amplitude degenerate orthogonal modes by a single feed point at an estimated location. By introducing some asymmetry in the structure the resonant frequencies of two degenerate modes can be made slightly different from each other and a phase difference of $90^{\circ}$ between the two amplitudes can be achieved [10]. The simplest way to split the fundamental $\mathrm{TM}_{11}$ mode is, cut a thin slit through the centre [5]. In this proposed antenna a hexagonal slot with unequal sides is chosen as the asymmetry or detuning element, in order to radiate circularly polarized waves. The radiation of circularly polarized wave is highly sensitive with respect to the position of the feed location and the dimensions of the central slot. The antenna is excited through co - axial probe at the location $\mathrm{X}$ to produce Right Handed Circular Polarisation (RHCP) and at the feed location Y to radiate electromagnetic waves with Left Handed Circular Polarisation (LHCP). The co-axial probe feed point for RHCP antenna is the mirror image as that for LHCP antenna with respect to the centre of the patch. The feed location is chosen along the locus of $50 \Omega$ characteristic impedance and is adjusted for good matching. The method of size reduction resorted to in this piece of work yields improved gain and axial ratio bandwidth compared to similar works in literature [1] to [10] and enunciated in Section IV.

\section{THEORY \& DESIGN}

\section{Theory of Circularly polarized Radiation}

- When a patch antenna is considered its mode impedance $\mathrm{Z}_{\mathrm{mn}}(\omega)$ may be expressed as in [5] 
Where $\mathrm{m}, \mathrm{n}=0,1 \ldots$, are the vertical and horizontal dimensions respectively. $R_{m n}$ represents the sum of the radiation, dielectric and metal losses, $\zeta_{\mathrm{mn}}(\omega)$ is the normalized frequency response written in terms of the quality factor $\mathrm{Q}_{\mathrm{mn}}$ and modal resonance frequency $\omega_{\mathrm{mn}}$. The quality factor $\mathrm{Q}$ depends to a large extent on the antenna geometry. By the proper selection of the feed point, the dominant mode is detuned into two equal amplitude orthogonal degenerate modes. Consider the antenna geometry where a single mode is detuned into two orthogonal modes $\mathrm{TM}_{\mathrm{e}}$ and $\mathrm{TM}_{\mathrm{o}}$, neglecting the effects of higher order modes. Here $\mathrm{TM}_{\mathrm{e}}$ is the orthogonal even mode and $\mathrm{TM}_{\mathrm{o}}$ is the orthogonal odd mode. Even mode is the mode that is symmetric with respect to the ' $\mathrm{Y}$ ' axis and odd mode is with respect to the ' $\mathrm{X}$ ' axis. Correspondingly total input impedance may be written as [5]

$$
\mathrm{Z}_{\text {in }}=\mathrm{Z}_{\mathrm{e}}+\mathrm{Z}_{\mathrm{o}}
$$

Where $Z_{\mathrm{e}}$ and $Z_{\mathrm{o}}$ are the corresponding even mode and odd mode impedances, $\omega_{\mathrm{e}}$ and $\omega_{\mathrm{o}}$ are the corresponding modal frequencies, with $\omega_{\mathrm{e}}<\omega_{\mathrm{o}}$. By introducing the asymmetry due to the irregular polygonal slot, the resonant frequencies of two degenerate modes are made slightly different from each other and a phase difference of $90^{\circ}$ between the two amplitudes is achieved. The normalized impedance ratio of odd to even mode is defined as in [5]

$$
\beta_{\zeta}(\omega)=\frac{\zeta_{0}(\omega)}{\zeta_{e}(\omega)}=\frac{1+\mathrm{jQ}\left(\frac{\omega}{\omega_{\mathrm{e}}}-\frac{\omega_{\mathrm{e}}}{\omega}\right)}{1+\mathrm{jQ}\left(\frac{\omega}{\omega_{\mathrm{o}}}-\frac{\omega_{\mathrm{o}}}{\omega}\right)}
$$

The angle of $\beta_{\zeta}(\omega)$ is the phase difference of the CP field components, while the magnitude is proportional to the axial ratio. Here the angle of $\beta_{\zeta}(\omega)$ is designed to be obtained as $90^{\circ}$ and the magnitude to be less than $3 \mathrm{~dB}$. The slot is introduced at the centre. As the fundamental mode exhibits a zero in the central area the fields will not be affected significantly. The fundamental mode splits into degenerate orthogonal modes by the proper choice of feed point and asymmetry. For the odd mode, which is oriented towards the slot ( $\mathrm{X}$ axis), the perturbation is minimum as the top and bottom width of the slot is made narrow enough. If the slot had been a rectangular geometry with width ' $b$ ' and height ' $d$ ' and no change in orientation, the odd mode surface current would not have been affected by this perturbation and the resonant angular frequency would have been approximately that of fundamental resonant frequency. Whereas, for the even mode (oriented about y axis), the surface current is forced to traverse more length around the slot. On account of more electrical length, even mode resonant frequency $\omega_{\mathrm{e}}$ is reduced. At this juncture, if a second orthogonal cut is made at the centre, it will enhance the electrical path of the odd mode and results in the reduction of corresponding resonant frequency. Here in this work, instead of making an orthogonal cut, the central width of the hexagonal slot is made larger than the top and bottom width. The same effect of second orthogonal cut is achieved due to this enhancement of central width. Just similar to the even mode, signal in odd mode also has to traverse more length and therefore resonant frequency is reduced. By selecting appropriate dimensions for the slot, the two degenerate mode frequencies are made nearly equal. Overall dimension of the antenna is reduced. But it was demonstrated in [11] that with annular shapes it is possible to enhance the bandwidth because less amount of energy is stored beneath the patch metallization and less quality factor therein. A mandatory condition for achieving circular polarization is equal amplitude and with a phase difference of $90^{\circ}$ for the degenerate modes [12].

\section{Design}

The design equations of the hexagonal patch antenna are derived in a similar approach as that of a circular patch. As the two shapes are equivalent to each other the equations for resonant frequency, effective radius of the circular patch are adopted as such[10]. The side length ' $a$ ' of the regular hexagon for a resonant frequency of $\mathrm{f}_{\mathrm{r}}=2.687 \mathrm{GHzis}$ designed as follows. The dominant mode resonance frequency of a circular patch is given by [11]

$$
\mathrm{f}_{\mathrm{r}}=\frac{X_{m n} c}{2 \pi \mathrm{r}_{\mathrm{e}} \sqrt{\epsilon_{r}}}
$$

where $\mathrm{f}_{\mathrm{r}}$ is the resonant frequency of the patch , $X_{m n}=1.8411$ for the dominant mode $\mathrm{TM}_{11}, c$ is the velocity of light in free space, $\boldsymbol{\varepsilon} r$ the relative permittivity of the substrate, ' $\mathrm{r}_{\mathrm{e}}$ ' is the effective radius of the equivalent circular patch and is given by [11]

$$
\mathrm{r}_{\mathrm{e}}=\mathrm{r}\left\{1-\frac{2 h}{\pi \mathrm{r} \varepsilon_{r}}\left(\ln \frac{\pi r}{2 h}+1.7726\right)\right\}^{0.5}
$$

where ' $r$ ' is the actual radius of the circular patch and ' $h$ ' is the

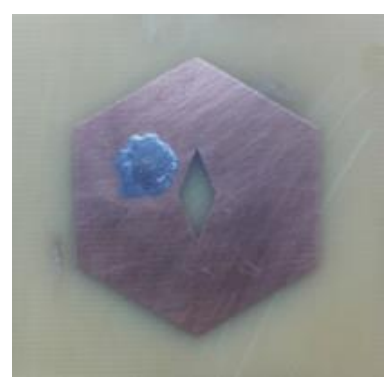

(a)

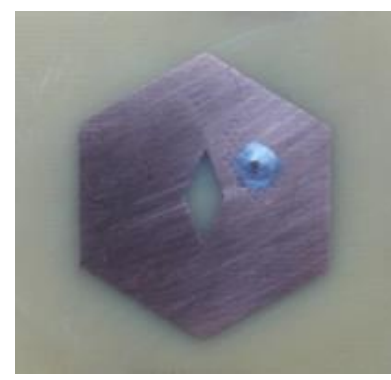

(b)
Fig. 2. Photographs of the antenna prototypes (a) RHCP (b) LHCP

height of the substrate. Then by equating the areas of a circle and a regular hexagon, the edge or side length of the regular hexagon 'a' may be found out as [11],

$$
\mathrm{a}=1.099 \mathrm{r}_{\mathrm{e}}
$$

By selecting proper slot dimensions and by choosing exact feed location, the two orthogonal degenerate modes are made nearly equal amplitude with a phase difference of $90^{\circ}$. Hence circularly polarized radiation is achieved. Overall size of the patch antenna is determined by the resonant frequency. The edge of the hexagonal patch is designed for a resonant frequency of $2.69 \mathrm{GHz}$. Due to the central slot the resonant frequency is reduced to $2.42 \mathrm{GHz}$ and thus the overall dimension is reduced by a factor of $22.5 \%$. 
The photographs of the proposed antennas are depicted in Fig. 2. To design the hexagonal patch, design equations are derived based on the geometry and frequency of operation after computing the guided wavelength $\lambda_{\mathrm{g}}$, that is the wavelength in the dielectric $\lambda_{\mathrm{g}}=\lambda_{0} / \varepsilon_{\mathrm{r}}$ eff, where $\lambda_{0}$ is the free space wavelength and the effective permittivity of the substrate $\varepsilon_{\mathrm{r} \text { eff }}$ is computed as 4.106 .

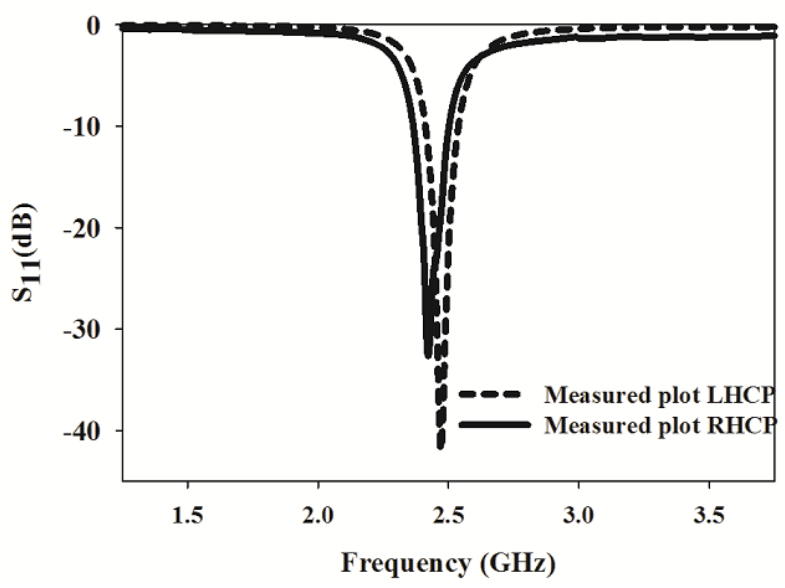

Fig. 3. Measured $\mathrm{S}_{11}$ plots of proposed RHCP and LHCP antennas

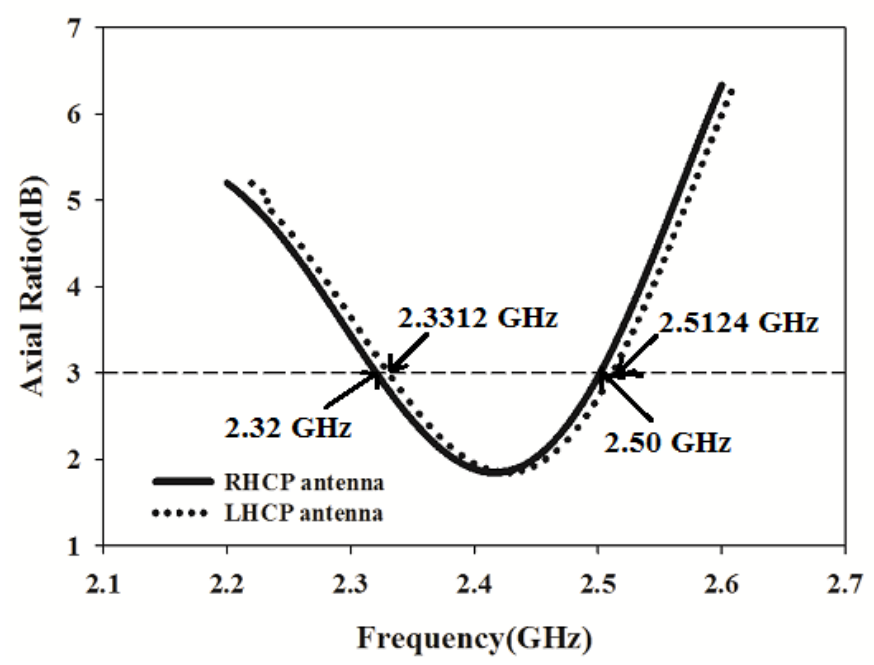

Fig. 4. Measured plots of axial ratio at $\mathrm{k}=1.62$.

\section{MEASURED RESULTS}

Using the computed parameters antenna was simulated using Ansoft HFSS 13.0. Optimum feed point was chosen for the best impedance matching of the antenna. Antenna prototype was fabricated on FR4 substrate with optimized parameters. The measured reflection characteristics of the proposed antennas are plotted in Fig. 3. It is noted that the fundamental resonant frequency of the antenna without slot is $2.687 \mathrm{GHz}$, whereas with slot, the measured return loss is $32 \mathrm{~dB}$ at $2.42 \mathrm{GHz}$ and the corresponding $10 \mathrm{~dB}$ bandwidth is $130 \mathrm{MHz}$ for the proposed
RHCP antenna. The measured return loss is $41.78 \mathrm{~dB}$ at 2.46 $\mathrm{GHz}$ for LHCP antenna with corresponding $10 \mathrm{~dB}$ impedance bandwidth of $125 \mathrm{MHz}$.

A parametric study is done by varying the parameter ' $\mathrm{k}$ ', which is the aspect ratio of height ' $d$ ' to the central width of the central irregular hexagonal slot, keeping ' $b$ ' constant. It is observed that when the value of $\mathrm{k}$ when exceeds 1.5 circularly polarized radiation is exhibited. The realization of circularly polarized radiation is confirmed at $\mathrm{k}=1.62$, as the necessary criterion of axial ratio $<3 \mathrm{~dB}$ is satisfied and depicted in Figure 4. When $\mathrm{k}=1.62$, the least simulated value of axial ratio $=$ $1.8212 \mathrm{~dB}$ is obtained with the fabricated prototype and the corresponding axial ratio bandwidth is calculated to be $180 \mathrm{MHz}(7.5 \%)$.
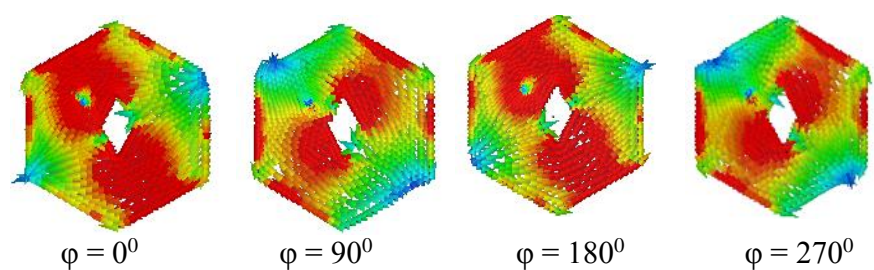

Fig. 5. Surface current distribution of RHCP antenna
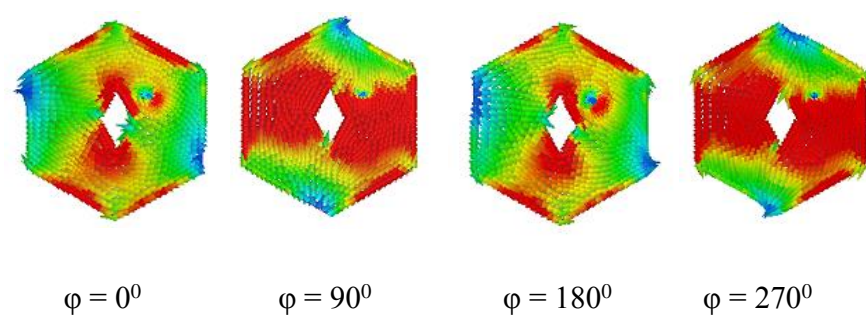

Fig. 6. Surface current distribution of LHCP antenna

The surface current distribution simulated at the centre frequency is plotted in Figure 5\&6 for RHCP and LHCP antennas respectively. Surface current distribution at $\varphi=0^{0}$ is equal in magnitude and opposite in direction to that at $\varphi=180^{\circ}$. Similar is the case of surface current distribution at $\varphi=90^{\circ}$ and at $\varphi=270^{\circ}$. Hence the criterion for circular polarization is satisfied. As the direction of view chosen as $+Z$-axis, the direction of rotation of current is clockwise and the sense of polarization is confirmed as left hand circular polarization (LHCP) for the feed position at Y. Feed position at X confirms right hand circular polarization (RHCP). Sense of direction of polarization of the fabricated antennas under study is tested using helical antennas designed at the measured resonant frequency $2.42 \mathrm{GHz}$ as per the design methodology in [13] \& [14]. The RHCP helical antenna gives more output when tested with RHCP under study and vice versa. 


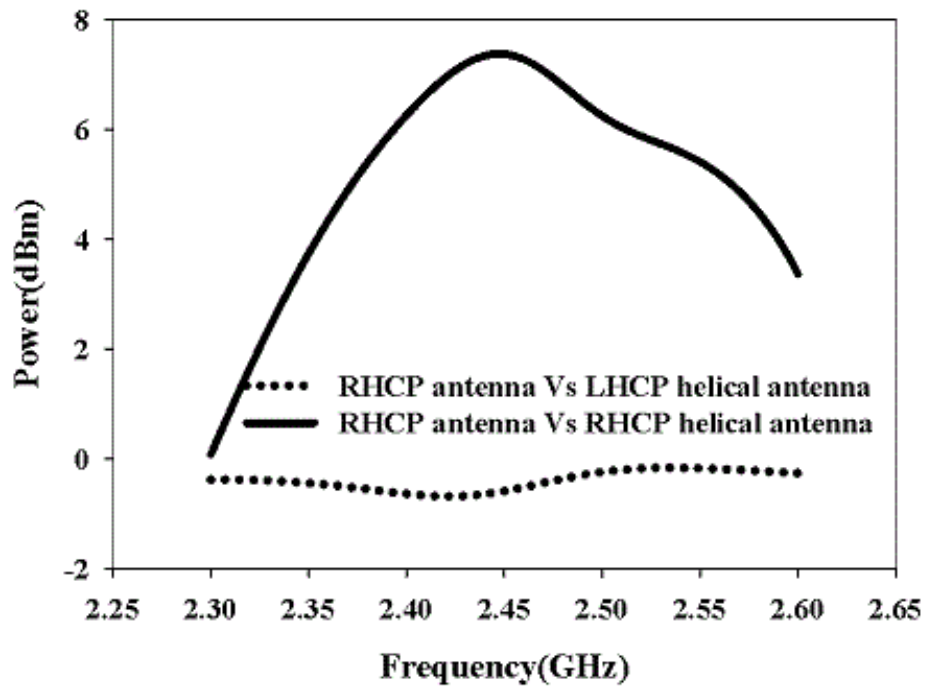

(a)

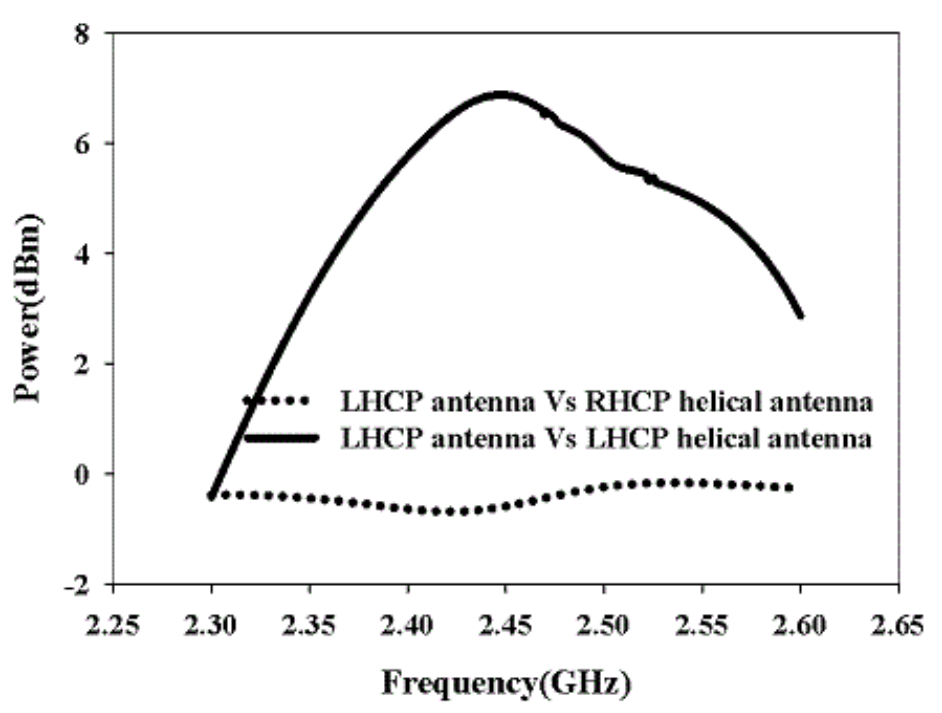

(b)

Fig. 7. S21 plots using helical antennas, when tested with (a) RHCP antenna (b) LHCP antenna

Graphs plotted in Figure 7 confirm the directions of sense of polarization of the LHCP and RHCP antennas under study Hexagonal shape is preferred for the antennas because it has more bandwidth compared to rectangular and circular patches [15].

Measured and simulated bore sight radiation patterns of RHCP and LHCP antennas are depicted in Figure 8 and found to be in good agreement. The comparison of measured values of the antenna is tabulated in Table I. More size reduction is due to the increased electrical length which is accounted by the shape of the patch and the slot. Increased axial ratio bandwidth is accounted by the choice of feed point and the aspect ratio of the slot. More impedance bandwidth is due to the choice of feed location and shape of the patch.

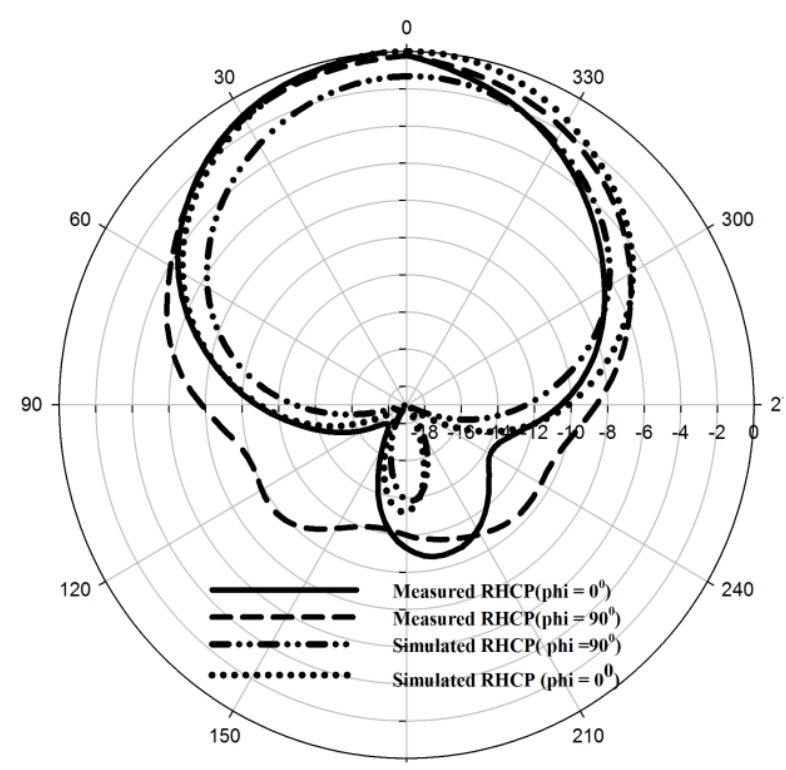

(a)

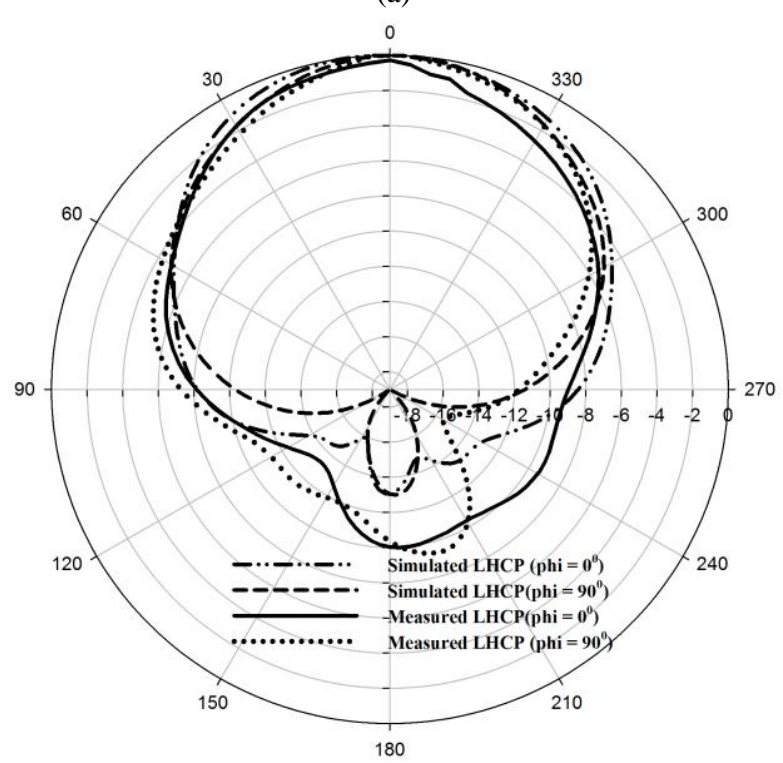

(b)

Fig. 8. Radiation pattern (a) RHCP antenna (b) LHCP antenna

The authors have experimentally proved in [14] that when the patch shape is dodecagonal gain is slightly less $(4.82 \mathrm{dBi}-$ $4.67 \mathrm{dBi}$ ), ARBW is $5.5 \%$ and when the shape is hexagonal with elliptical and circular slots the gain is $4.5 \mathrm{dBi}$ and ARBW is $5.8 \%$ [15]; both designed for the same resonant frequency $2.45 \mathrm{GHz}$.

TABLE I

COMPARISON OF MEASURED VALUES

\begin{tabular}{|l|c|c|c|}
\hline Parameter & $\begin{array}{c}\text { Circular } \\
\text { patch antenna }\end{array}$ & $\begin{array}{c}\text { Measured } \\
\text { value } \\
\text { (RHCP } \\
\text { antenna) }\end{array}$ & $\begin{array}{c}\text { Measured } \\
\text { value } \\
\text { (LHCP } \\
\text { antenna) }\end{array}$ \\
\hline
\end{tabular}




\begin{tabular}{|l|c|c|c|}
\hline & $\begin{array}{c}\text { with elliptical } \\
\text { slot [5] }\end{array}$ & & \\
\hline $\begin{array}{l}\text { Center } \\
\text { frequency }\end{array}$ & $2.45 \mathrm{GHz}$ & $2.42 \mathrm{GHz}$ & $2.46 \mathrm{GHz}$ \\
\hline Return loss & $19 \mathrm{~dB}$ & $32 \mathrm{~dB}$ & $42 \mathrm{~dB}$ \\
\hline Gain & $3.85 \mathrm{dBi}$ & $4.9 \mathrm{dBi}$ & $4.91 \mathrm{dBi}$ \\
\hline $\begin{array}{l}\text { Minimum axial } \\
\text { ratio }\end{array}$ & - & $1.8212 \mathrm{~dB}$ & $1.815 \mathrm{~dB}$ \\
\hline $\begin{array}{l}\text { Axialratio } \\
\text { bandwidth }\end{array}$ & $1.22 \%$ & $7.43 \%$ & $7.365 \%$ \\
\hline $\begin{array}{l}\text { 10dBimpedance } \\
\text { bandwidth }\end{array}$ & $5.3 \%$ & $5.4 \%$ & $5.1 \%$ \\
\hline $3 \mathrm{~dB}$ beamwidth & - & $80^{0}$ & $86^{0}$ \\
\hline Size reduction & $9.5 \%$ & $22.5 \%$ & $22.5 \%$ \\
\hline
\end{tabular}

[3] Chen W.S., C.K Wu.andK.L.Wong, Novel compact circularly polarized square microstrip antenna, IEEE Transactions on Antennas and Propagation, Vol.49, No.3,pp 340-342, 2001

[4] Pomsathit A., C.Benjangkaprasert, N.Anantrasirichail, V.Chutchavong, and T.Wakabayashi, Circularly Polarized Right Angle Slot Antennas for WLAN of IEEE 802.llb/g, International Symposium on Communications and Information Technologies ISCIT 2008, Lao, pp 46-50,2008.

[5] Maddio Stefano, Alessandro Cidronali Gianfranco Manes, A New Design Method for Single-Feed Circular Polarization Microstrip Antenna With an Arbitrary Impedance Matching Condition, IEEE Transactions on Antennas and Propagation, Vol.59 , No.2, pp 379-389, 2011.

[6] Barbero J., H. Lazo, F. Municio, and M. TeDeCe, Model for the patch radiator with a perturbation to achieve circular polarization, IEE Colloquiumon Recent Developments in Microstrip Antennas, London, , pp 6/1 - 6/4., Feb 1993

[7] Horng-Dean Chen, Shang-Huang Kuo, Chow-Yen- Desmond Sim, Ching-Han Tsai, Coupling-Feed Circularly Polarized RFID Tag Antenna Mountable on Metallic Surface IEEE Transactions on Antennas and Propagation, Vol. 60, No. 5, 2012.

\section{Appropriateness of the antenna for RFID reader applications at $2.45 \mathrm{GHz}$}

The RHCP antenna prototype offers a $10 \mathrm{~dB}$ impedance bandwidth of $130 \mathrm{MHz}$ (2.3645 GHz- 2.4965), $3 \mathrm{~dB}$ ARBW of $180 \mathrm{MHz}$ ( $2.32 \mathrm{GHz}-2.50 \mathrm{GHz})$ and a gain of $4.9 \mathrm{dBi}$. Similarly the LHCP antenna prototype offers a $10 \mathrm{~dB}$ impedance bandwidth of $125 \mathrm{MHz}(2.5415 \mathrm{GHz}-2.4165 \mathrm{GHz})$ and $3 \mathrm{~dB}$ ARBW of $181.2 \mathrm{MHz}(2.3312 \mathrm{GHz}-2.5124 \mathrm{GHz})$ and a gain of $4.91 \mathrm{dBi}$. These values are in compliance with standards of parameters for air interface communications for RFID applications at $2.45 \mathrm{GHz}$ [16].

\section{CONCLUDING REMARKS}

A novel design of a single feed circularly polarized regular hexagonal patch antenna with irregular hexagonal slot has been designed, simulated and experimentally investigated. The introduction of central hexagonal slot perturbation method is found to be effective. The antennas provide stable symmetrical patterns with wide angle half power beam widths, good impedance bandwidths circular polarization characteristics and reasonable peak gain values. The antennas are compact, simple to design cost effective and suitable for wide angle coverage RFID reader applications at $2.45 \mathrm{GHz}$.

\section{ACKNOWLEDGMENTS}

The authors acknowledge University Grants Commission (UGC), Government of India, for providing the teacher fellowship under the Faculty Development Programme.

\section{REFERENCES}

[1] Horng-Dean Chen, Shang-Huang Kuo, Chow-Yen-Desmond Sim, and Ching-Han Tsai Coupling-Feed Circularly Polarized RFID Tag Antenna Mountable on Metallic Surface, IEEE Transactions On Antennas And Propagation, Vol. 60, No. 5, May 2012.

[2] Row J.S. and C.Y.Ai, Compact design of single feed circularly polarized microstrip antenna, Electronics Letters, Vol.40, No.18, pp 1093-1094, 2004.

[8] K.C.Prakash, S.Mathew, R.Anitha, P.V. Vinesh, M.P.Jayakrishnan, P.Mohanan, K. Vasudevan, Circularly polarized dodecagonal Patch Antenna with Polygonal slot for RFID Applications Progress in electromagnetic Research, vol.61, 9-15,2016.

[9] Prakash K.C, Vinesh P.V, Jayakrishnan. M.P, Dinesh. R, Mohammad Ameen, Vasudevan K, Hexagonal Circularly Polarized Patch Antenna for RFID Applications, Int. Journal. Cybernetics and Informatics, vol. 5, no. 2, pp. 173-182, 2016

[10] Horng-Dean Chen, Shang-Huang Kuo, Chow-Yen- Desmond Sim, Ching-Han Tsai, Coupling-Feed Circularly Polarized RFID Tag Antenna Mountable on Metallic Surface, IEEE Transactions on Antennas and Propagation, Vol. 60, No. 5, 2012.

[11] Kushwaha Nagendra and Raj Kumar, Design Of Slotted Ground Hexagonal Microstrip Patch Antenna And Gain Improvement With FSS Screen, Progress In Electromagnetics Research B, Vol. 51, pp 177-199, 2013.

[12] A. Bhattacharyya and L. Shafai, A wider band microstrip antenna for circular polarization, IEEE Transactions on Antennas and Propagation, Vol. 36, No. 2, pp 157-163, 1988.

[13] Steven (Shichang) Gao, Qi Luo and Fuguo Zhu," Circularly Polarized Antenna”, John Wiley \& Sons Ltd, United Kingdom, 2014.

[14] Balanis C. A., " Antenna theory: Analysis and design, second edition", John Wiley \& Sons Inc, New Delhi,2007.

[15] Mathur V., Manisha Gupta Comparison of Performance Characteristics of Rectangular, Square and Hexagonal Microstrip Patch Antennas, In Proceedings of 3rd International Conference on Reliability, Infocom Technologies and Optimization (ICRITO) (Trends and Future Directions), Noida ,India, October2014

[16] www.iso.org

[17] Nasimuddin, X. Qing, and Z. N. Chen, "Compact asymmetricslit microstrip antennas for circular polarization," IEEE Trans. Antennas Propag., vol. 59, no. 1, pp. 285-288, 2011.

[18] Nasimuddin, X. Qing, and Z. N. Chen, "Compact circularly polarized symmetric-slit microstrip antennas," IEEE Antennas Propag. Mag., vol. 53, no. 4, pp. 63-75, 2011.

[19] Y.-F. Lin, H.-M. Chen, S.-C. Pan, Y.-C. Kao, and C.-Y. Lin, "Adjustable axial ratio of single-layer circularly polarised patch antenna for portable RFID reader," Electron. Lett., vol. 45, no. 6, p. 290, 2009.

[20] R. Caso, A. Michel, M. R. Pino, and P. Nepa, "Dual-band UHFRFID/ WLAN circularly polarized antenna for portable 
RFID readers," IEEE Trans. Antennas Propag., vol. 62, no. 5, pp. 2822-2826, May 2014.

[21] Qiang Liu, Junyu Shen, Jungang Yin, Member, IEEE, Hongli Liu, and Yuanan Liu, Member, IEEE, Compact 0.92/2.45-GHz Dual-Band Directional Circularly Polarized Microstrip Antenna for Handheld RFID Reader Applications IEEE TRANSACTIONS ON ANTENNAS AND PROPAGATION, VOL. 63, NO. 9, SEPTEMBER 20153849

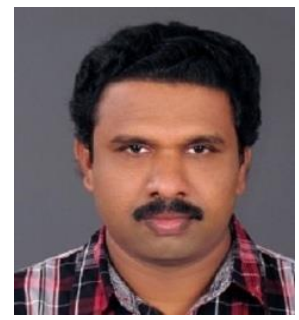

Prakash K.C passed his M.Sc Degree in Electronics Science from Cochin University of Science and Technology, Kerala, India, in 1993, with third rank. He has got 22 years of teaching experience in Electronics and holds the designation of Associate Professor in in Sree Ayyappa College, Eramallikkara; a Kerala Govt. Aided college affiliated to the University of Kerala Currently he is engaged in research under the faculty development programme of UGC, in the area of microwave antennas for RFID applicatios. He has got 1 international ,1 national journal and four international conference publications to his credit. He is a Fellow of IETE. His areas of interest include RFID CP antennas, metamaterial, energy harvesting etc.

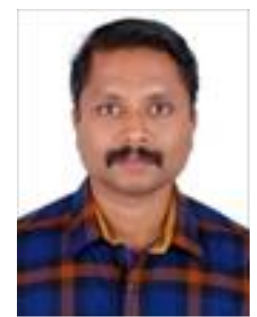

Vinesh $\mathbf{P} \mathbf{V}$ received the B.Sc. degree in electronics from the University of Kannur, India, and the M.Sc. degree in Electronics from the MES College Erumely, Kottayam, India, in 2004 and 2006,respectively. He is currently working towards the Ph.D. degree at Cochin University of Science and Technology (CUSAT), Kochi, India. His research interests include designing of multiband antennas, planar inverted $\mathrm{F}$ antennas, ZOR antenna etc.

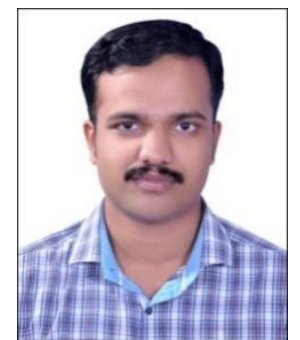

Vivek $\mathbf{R}$ received the B.Sc. degree and the M.Sc. degree in electronics from University of Calicut, India, in2008 and 2010, respectively. $\mathrm{He}$ is currently working towards the Ph.D. degree at Cochin University of Science and Technology (CUSAT), India. His research interests include designing of multiband antenna, folded/loop antenna, PIFAantennas, chiplessRFIDs, metaresonators and UWB

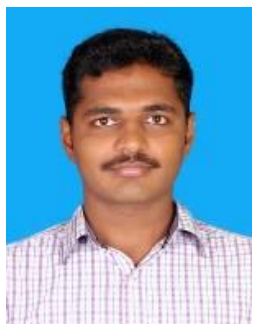

Mohammad Ameen received his B.Tech degree and M.Tech degree in Electronics and Communication Engineering from Mahatma Gandhi University, Kerala, in 2012 and 2014, respectively. He has 1 year of research experience in Microwave field. Currently he is a Senior Research Fellow in CSIR (New

Delhi) funded project at Department of Electronics, Cochin University of Science and Technology, Cochin, Kerala. He has got one international journal and four international conference publications to his credit. His research interests include high gain antennas and MIMO antennas.

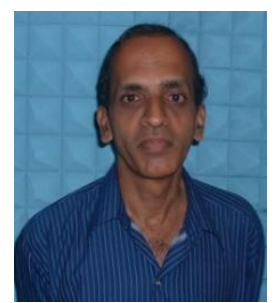

Vasudevan K. took $\mathrm{PhD}$ in 1982 in Microwave antennas and joined as lecturer in Cochin University of science and technology in 1985 . He is a professor in this University since 1995. He was head of department of Electronics from 2004 to 2010 and Dean, faculty of technology during 2010 to 2013. Currently, he is a CSIR Emeritus scientist in Cochin University. He has more than 220 publications in International journals and conference proceedings and has more than 1200 citations and an h- index of 19. $\mathrm{He}$ has been an investigator for about 20 major projects from various agencies having a total outlay of 30 crores of rupees. He has chaired and presented papers in several IEEE international conferences abroad.. He is a senior member of IEEE and Fellow of IETE. 